\title{
Web 2.0: una herramienta en la enseñanza universitaria
}

Fecha de recibido: 6 de abril de 2015

\section{RESUMEN}

Este estudio reúne una serie de experiencias educativas usando la Web 2.0, con lo cual se confirma la utilidad de las Tecnologías de la Información y Comunicación (TIC), en la enseñanza de la asignatura Investigación Aplicada. Sin embargo, según la amplia aceptación mostrada por los estudiantes, estas, pueden ser utilizadas en cualquier asignatura que se imparta a nivel universitario. Se trabajó con 12 estudiantes de la carrera Física-Matemática $\mathrm{V}$ año, utilizando de forma sistemática herramientas tecnológicas como: Facebook, Dropbox, Survey Monkey, Google URL Shortener, Microsoft Word, Netquest, Statstm y Citation Machine. Para obtener la valoración de estas herramientas, se utiliza una encuesta estructurada con un Alfa de Cronbach de 0.71, contrastándola con los escritos dejados por los partícipes en la página de Facebook "Investigación Matemática". Además, se evidencian los alcances de los medios tecnológicos cuando se usan correctamente en el proceso educativo, y la importancia que tiene el desempeño docente en la gestión del proceso de enseñanza.

\section{Palabras clave}

Web 2.0, innovación, herramientas tecnológicas.
Jairo José Flores Morales Departamento de Ciencias de la Educación y Humanidades Universidad Nacional Autónoma de Nicaragua, Managua jairofmdjmix@yahoo.com José Eligio Guzmán Contreras
de la Educación y Humanidades Universidad Nacional Autónoma de Nicaragua, Managua eligioinach@ hotmail.com

Fecha de aprobado: 12 de julio de 2015

\section{ABSTRACT}

This study gathers a number of educational experiences using Web 2.0, with which is confirmed the usefulness of the Technologies of the Information and Communication (TIC), in teaching Applied Research course. Nevertheless, according to the wide acceptance shown by the students, these can be used on any subject that is given to university level. We worked with 12 students of Physics-Mathematics career, from fifth level, making systematic use of technological tools such as: Facebook, Dropbox, Survey Monkey, Google URL Shortener, Microsoft Word, Netquest, Statstm and Citation Machine. To obtain the valuation of these tools, a structured questionnaire with Cronbach's alpha of 0.71 is used, contrasting with the writings left by the participants in the Facebook page "Mathematical Research". Furthermore, the scopes of the technological means are evident when they are used correctly in the educational process, and the importance of teacher performance in managing the teaching process.

Key words

Web 2.0 innovation, technological tools. 


\section{INTRODUCCIÓN}

Constantemente se escucha entre los estudiantes hablar de Facebook, Twitter, Instagram, Tango, Wassap, entre otros; evidencia del constante intercambio de información que fluyen en diversas plataformas virtuales, todo esto obliga a preguntarnos: ¿Los docentes estamos aprovechando correctamente los recursos que nos ofrece internet?, ¿somos conscientes de la era digital en que nos encontramos inmersos? Como educadores, se hace evidente la necesidad en ser partícipes de ese cambio que la globalización tecnológica ha traído consigo.

En este sentido, los sistemas educativos en su afán de mejorar la educación para las masas sociales, han venido utilizando una serie de recursos tecnológicos que se encuentran disponibles en los espacios virtuales, empezando a transformar simples comunidades sociales, en comunidades de práctica y aprendizaje, donde el conocimiento teórico y muy especialmente el práctico adquieren mucha importancia (López, 2007).

Estudios previos demuestran la importancia de las herramientas tecnológicas en diversos aspectos como la motivación, las comunidades de práctica, transmisión del conocimiento, trabajo colaborativo y la asimilación de aprendizajes, tal como lo indica García (2008), Robles (2009), Domínguez (2009), Chavarro (2010) y Pisticelli (2010), citados por Barajas y Álvarez (2013). Estos estudios confirman que las herramientas tecnológicas en educación permiten variadas alternativas de aprendizaje, diversas formas didácticas y metodológicas que propician la creatividad en el estudiante.

La web 2.0 es el nombre que se le ha asignado al conjunto de herramientas que se encuentran disponibles en la web actual: interactiva y colaborativa. Estas herramientas se caracterizan por las posibilidades que ofrecen a los usuarios de tener un doble rol: ser protagonistas y a la vez público de la información que circula por la web, destacándose algunas de ellas, por estar enfocadas desde una perspectiva pedagógica.

Esta filosofía de compartir y de participar en grupo para la elaboración de diversos recursos o documentos, para el proceso de enseñanza - aprendizaje, permite tanto al alumnado como al profesorado adaptarse a la distintas situaciones que nos proporciona el sistema educativo. Sin embargo, para lograrlo se deben tener presente una serie de aspectos antes de implantar la Web 2.0 dentro del proceso de enseñanza - aprendizaje. 
Primeramente se debe cambiar el rol que tiene el educador y el educando, pasar de orador de conocimientos a organizador y orientador de información, permitiendo que el alumno participe activamente en su propio aprendizaje. En segundo lugar está la motivación constante de la clase, y la generación de nuevos estilos de aprendizaje que generen competencias nuevas en ellos (Zamarrazo y Amorós, 2011).

Por otro lado, en nuestro contexto, pocos docentes recurren a esta herramienta tecnológica para utilizarla en las aulas. Aún existen docentes que huyen a este tema o evaden diciendo que la forma de enseñar debe ser la misma que hace varios años. Señalamiento que contradice la afirmación, de que la educación siempre se ha adaptado a los cambios tecnológicos existentes.

Se necesita entonces, tener la fuerza de voluntad para desechar el paradigma de la enseñanza tradicional, y reemplazarlo o reformarlo para poder conectar nuestras asignaturas con el mundo real, por medio de la tecnología.

\section{MATERIALES Y MÉTODOS}

Partiendo del diagnóstico realizado en el año 2013, a los estudiantes universitarios de la Facultad Regional Multidisciplinaria de Chontales, el cual muestra que la red social que más utilizan los estudiantes es Facebook, representando un $80 \%$, con un amplio margen con relación a Twitter que aparece en segundo lugar con un $8 \%$ (Flores, 2014). Y tomando como base los resultados de la experiencia realizada con estudiantes de Ingeniería Agroindustrial y Administración de Empresas ese mismo año, al utilizar la página en Facebook llamada "Mateayuda". Se decide entonces darle continuidad a esta idea, por lo que se crea una nueva página en Facebook llamada "Investigación Matemática" para ser usada en la asignatura Investigación Aplicada con los estudiantes de V año en la carrera Física-Matemática.

Para la implementación y uso de la página en la asignatura, se utilizan además, herramientas propias de la web 2.0, tales como: Dropbox, Survey Monkey, Google URL Shortener, Microsoft Word, Netquest, Statstm y Citation Machine, se brindaron mini talleres para que se dominaran esos medios digitales.

Este trabajo, por sus características tiene un enfoque mixto, es cualitativo ya que estudia la realidad en su contexto natural, tal y como sucede, interpretando los fenómenos de acuerdo con los significados que tienen para las personas implicadas, además, produce datos descriptivos como 
las propias palabras de las personas, habladas o escritas, y la conducta observable. Es a la vez cuantitativo, porque permite examinar los datos de manera numérica, especialmente en el campo de la Estadística, haciendo demostraciones con los aspectos separados de su todo, a los que se asigna significado numérico y hace inferencias.

Por otro lado, el empleo de ambos procedimientos, cuantitativos y cualitativos, ayuda a corregir los sesgos propios de cada método (Cook y Retechardt, 2004). Además, el utilizar las fortalezas de ambos tipos de investigación combinándolas y tratando de minimizar sus debilidades potenciales, permiten que la recolección, análisis y vinculación de datos cualitativos y cuantitativos en un mismo estudio ofrezcan mejores resultados. (Hernández, Fernández y Baptista, 2010)

Se trabaja con 12 estudiantes que forman el total de alumnos de la sección, resultando provechoso utilizar todo el universo de estudiantes, debido a la modalidad por encuentros en que se realiza la experiencia. A todos los partícipes se les atiende online, evitando así, que los estudiantes viajaran para ser tutorados presencialmente, disminuyendo así los gastos económicos de los mismos.

Para obtener las valoraciones de los estudiantes, en relación a la implementación de la web 2.0 en el proceso de enseñanza-aprendizaje, se utiliza una encuesta estructurada con 20 ítems.

Para comprobar la consistencia interna del instrumento elaborado, se utilizó el coeficiente Alfa de Cronbach, basado en el promedio de las correlaciones entre los ítems. La validez de un instrumento se refiere al grado en que el instrumento mide aquello que pretende medir.

De acuerdo a George y Mallery (2003), la fiabilidad se relaciona con el hecho de que el instrumento de medición produzca los mismos resultados cada vez que sea administrado a la misma persona y en las mismas circunstancias. Obteniéndose un alfa de Cronbach de 0.71, aseverando así, que la consistencia interna de los ítems es aceptable.

Se utiliza el procesador de datos estadísticos llamado Startical Product and Service Solutions, por sus siglas en inglés (SPSS), en su versión 21. Además, se hace uso del paquete Office para la presentación de los resultados. 


\section{RESULTADOS}

La puesta en marcha de la página creada en Facebook, y las diversas herramientas que ofrece la web 2.0, permiten una mayor aceptación de la asignatura por parte de los estudiantes.

Las opiniones de estos, permiten conocer qué tanto les agradó recibir clase de matemática, usando una plataforma virtual gratuita como lo es Facebook.

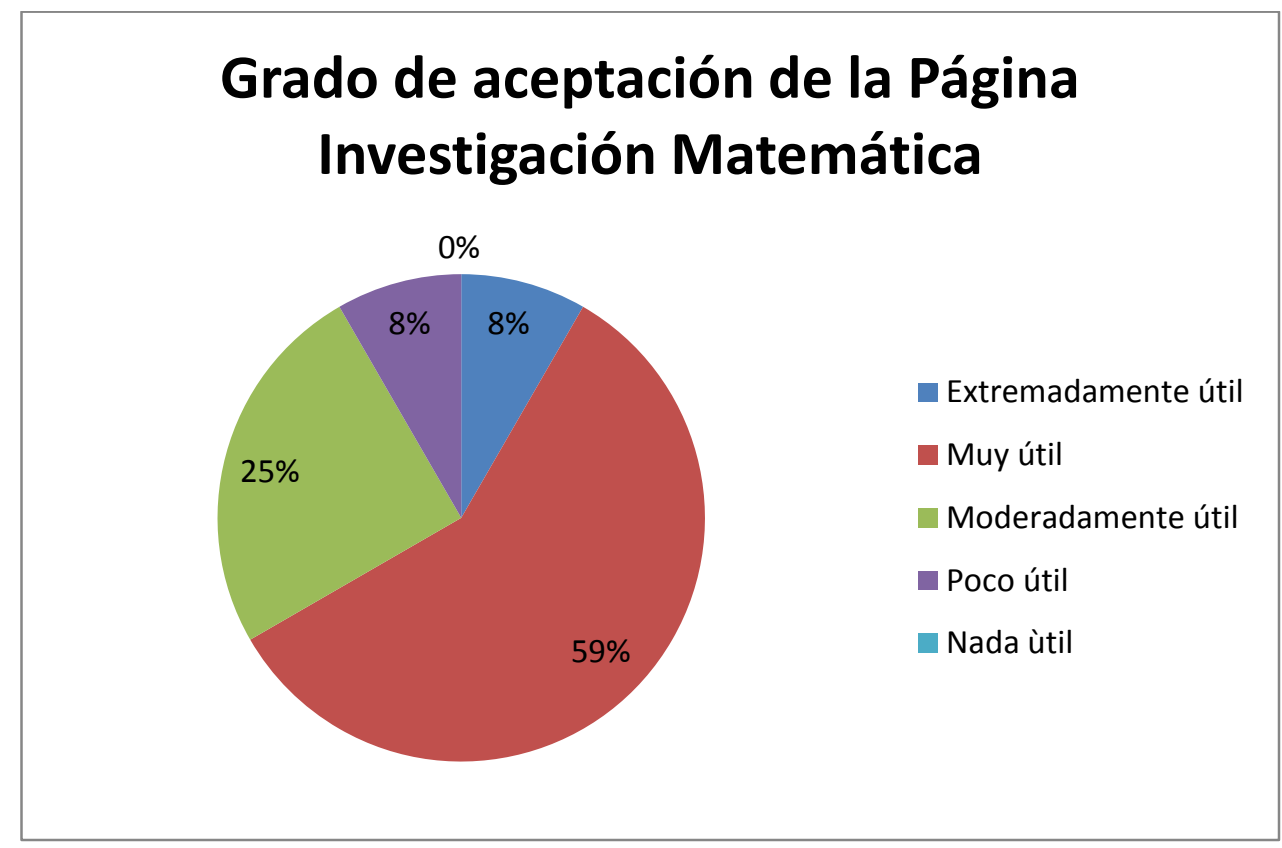

Figura 1: Utilidad de la página para los estudiantes.

La figura 1, refleja que la página fue considerablemente aceptada por los estudiantes, más del 90\% la definió de esa manera. Nuevamente se vuelve a ratificar lo encontrado en Flores(2014), sobre el uso de Facebook en materia educativa, los estudiantes opinan que el papel de esta red social les favorece en gran medida para reforzar aprendizajes, comunicarse con el tutor, envío de comentarios y para conocer información propia de la asignatura. 


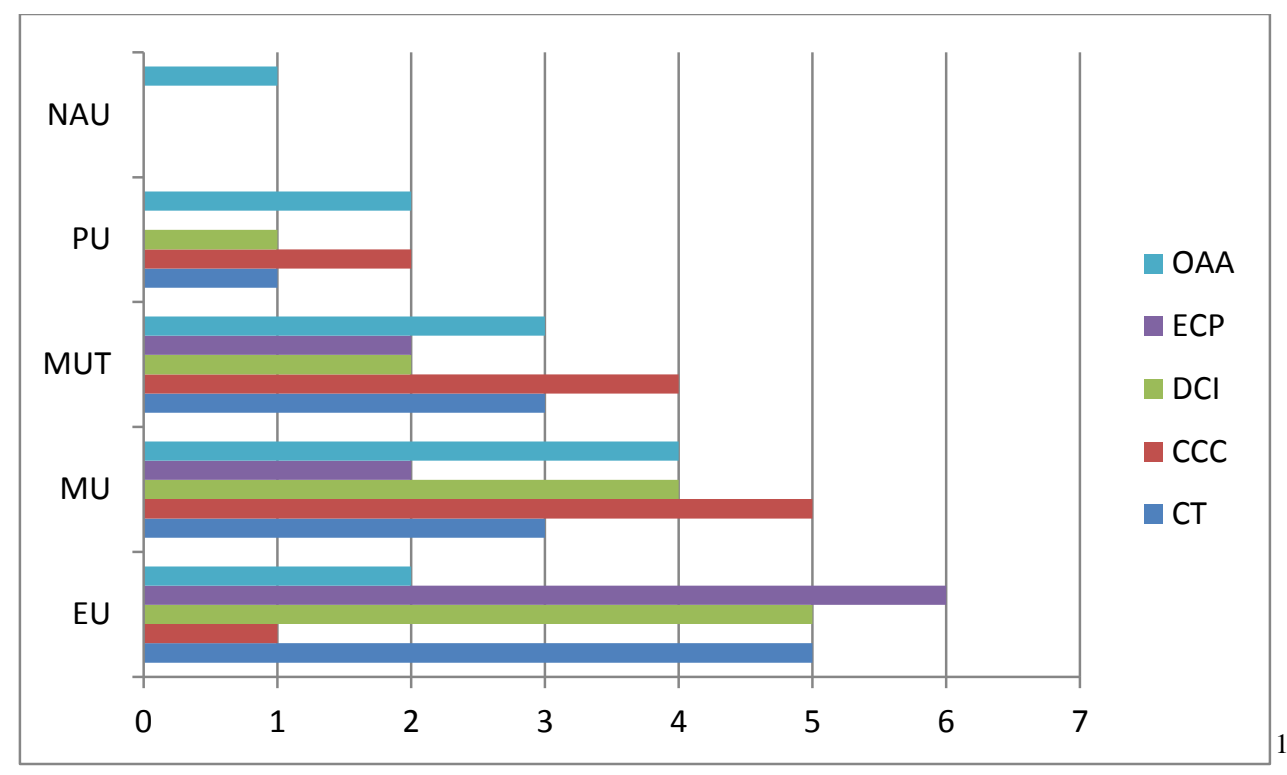

Figura 2: Papel desempeñado por Facebook.

Claramente se aprecia que a los estudiantes les agrada experimentar con herramientas tecnológicas que salen de la rutina. La originalidad, innovación e interacción entre el estudiante y tutor, son aspectos positivos valorados por los estudiantes cuando hicieron uso de la página, enfocándose en la parte interactiva y comunicativa que Facebook posee (Ver figura 2).

No se puede finalizar la disciplina sin antes valorar el propio desempeño del docente, siendo esto un acto obligatorio para los que reflexionamos sobre nuestra praxis, y medimos los aciertos y desaciertos que suscitan en el desarrollo de la asignatura.

La figura 3 evidencia la alta valoración del desempeño del docente, lo que indica que las estrategias y herramientas utilizadas por el tutor, llenaron las expectativas de sus estudiantes.

Una de las herramientas mejor valoradas son los videos tutoriales realizados por el docente, ya que estos, permiten retroalimentar los conocimientos del estudiantado y ayudar a aquellos que poseen dificultades en el uso de la tecnología. Estas herramientas tecnológicas utilizadas en la enseñanza de la asignatura, resultaron ser pieza fundamental en el logro de objetivos.

\footnotetext{
${ }^{1}$ OAA: Otras actividades académicas; ECP: Envío de comentarios o preguntas; DCI: Dar a conocer información de la asignatura; CCC: Comunicación entre Compañeros de Clase; CT: Comunicación con el tutor. EU: Extremadamente Útil; MU: Muy Útil; MUT: Moderadamente Útil; PU: Poco Útil; NAU: Nada Útil.
} 
Sin embargo, el uso de Dropbox facilita la forma en que se comparten archivos, sin necesidad de usar correos electrónicos. Por lo que cada grupo de estudiantes, tienen su propia carpeta en la que el tutor y los estudiantes comparten información propia de la asignatura.

\section{¿Cuál es tu valoración con relación al trabajo de tu tutor?}

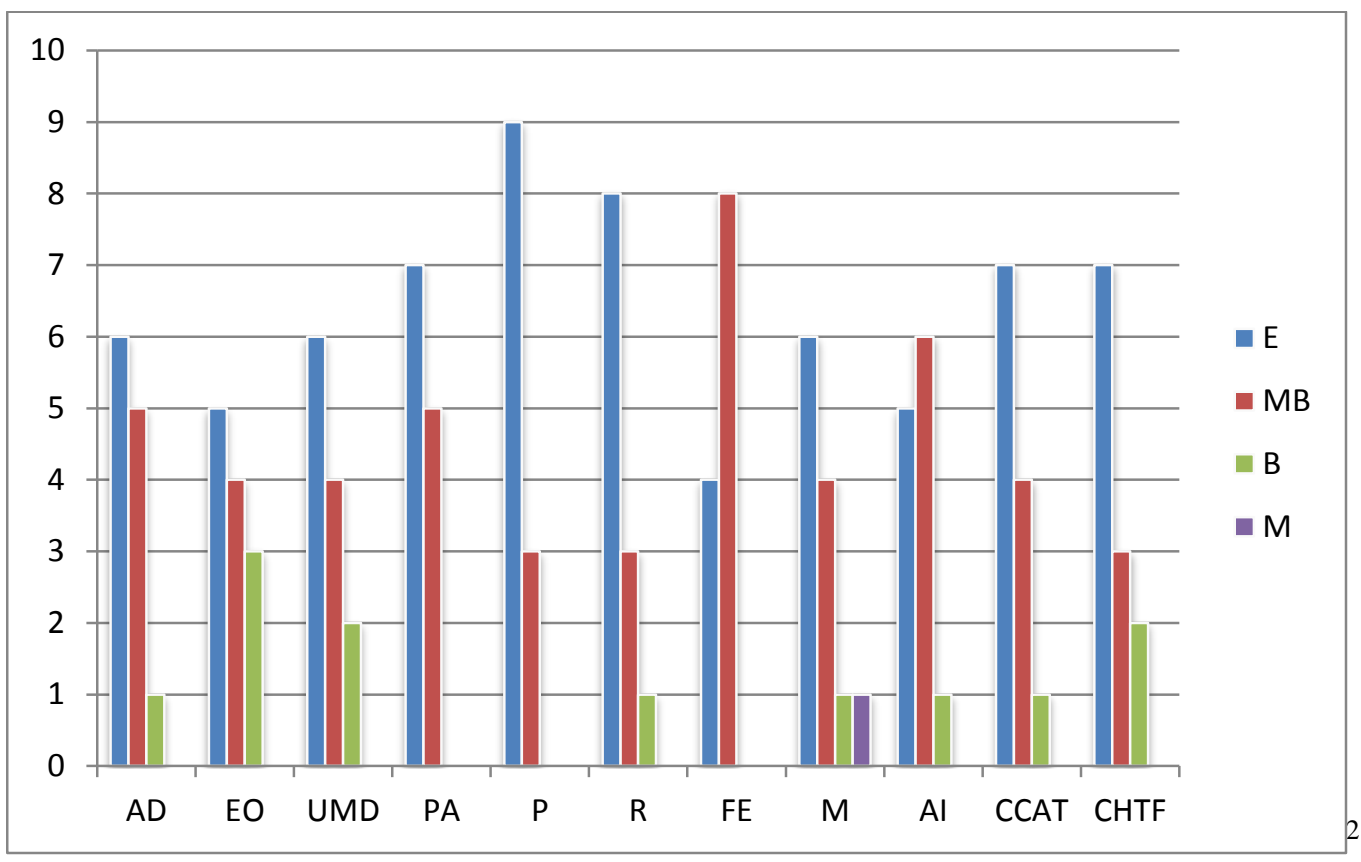

Figura 3: Valoración del trabajo del tutor por los estudiantes.

Fácilmente se analizaban los avances de los estudiantes y sobre todo si habían incluido las recomendaciones dejadas en borradores anteriores, esto disminuía el tiempo y costo económico para los estudiantes, ya que aún existen casos en que los docentes mandan a imprimir los avances en físico, los revisan y luego los estudiantes tienen que mejorar dichas revisiones, repitiéndose ese ciclo agotador para los alumnos (Ver figura 4).

${ }^{2}$ E: Excelente; MB: Muy Bueno; B: Bueno; M: Malo.

AD: Atención a tus dudas; EO: Explicación Objetiva; UMD: Uso de medios digitales; PA: Porte y aspecto; P: Puntualidad; R: Responsabilidad; FE: Forma de Evaluar; M: Motivación; AI: Atención Individual; CCAT: Claridad en las Correcciones de los Avances en tu Trabajo; CHTF: Correcciones Hechas en Tiempo y Forma. 

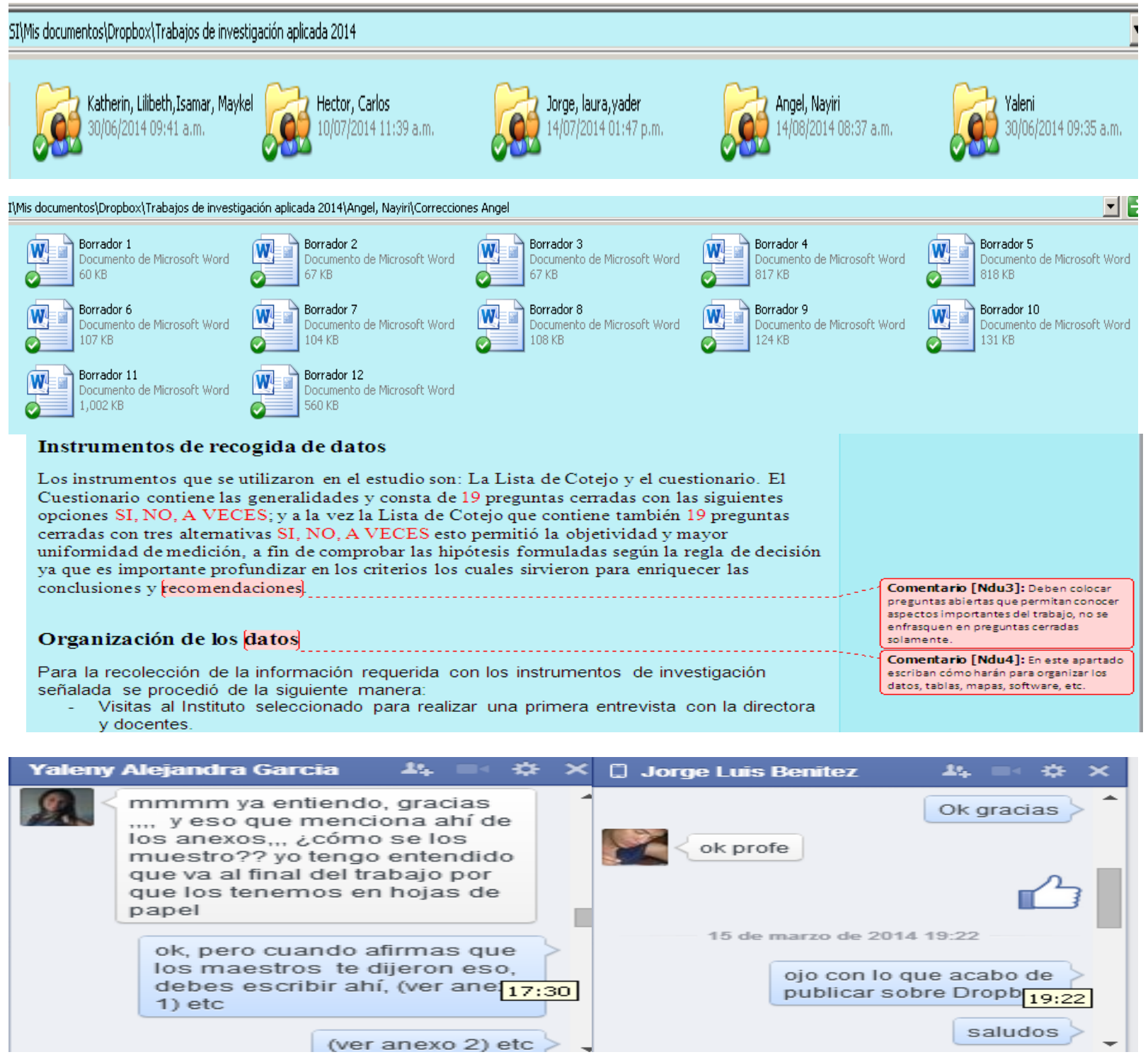

Figura 4: Intercambio de archivos a través de carpetas en Dropbox, Correcciones elaboradas en Microsoft Word, Avances en el trabajo investigativo por parte de un grupo de estudiantes y Comunicación Instantánea entre el tutor y los estudiantes.

La comunicación entre los estudiantes y su docente está siempre cargada de cordialidad y en búsqueda de responder dudas sobre el trabajo investigativo. Esta forma de comunicación contrasta con la forma tradicional de hacerlo en forma presencial, haciendo viajar a los estudiantes desde otros departamentos por una hora de tutorías, pudiendo hacerse más fácil de esta manera.

Esto constata que la Web 2.0 permite cambiar la forma en que damos clases, la infinidad de recursos que en ella se encuentran, favorece que dejemos paulatinamente lo rutinario por lo 
novedoso, lo tradicional por lo innovador, la educación presencial por una a distancia en forma on-line.

Esta experiencia verifica que la innovación tecnológica puede ir acorde con la innovación pedagógica, la amplia variedad de utilidades que ofrece la Web 2.0, permite crear formas de enseñar muy diferentes a las que estamos acostumbrados a realizar. La gestión y el adecuado uso didáctico de estos medios, mejoran el proceso de enseñanza aprendizaje de cualquier asignatura, más aún, si esta se ofrece en modalidades de profesionalización.

\section{DISCUSIÓN DE LOS RESULTADOS}

Las nuevas herramientas alternativas que ofrece la Web 2.0, permiten disminuir la brecha existente entre enseñanza y calidad de los aprendizajes. Aspecto que toma mayor relevancia con nuestros estudiantes que viven inmersos en la era digital, llenos de motivación por aprender en forma diferente, usando éstas tecnologías.

Tanto el contexto cambiante, como las nuevas metodologías presentes en la actualidad, permiten al docente implementar actividades que se contraponen con el enfoque tradicional que envuelve en muchos casos al sistema educativo universitario. Con este estudio, se verifica que las TIC's fortalecen la enseñanza en las Universidades, a los estudiantes les agrada usar plataformas virtuales para reforzar sus aprendizajes y sobre todo, les motiva a seguir aprendiendo de una forma interesante para ellos.

El amplio rol que juega el docente es relevante desde dos perspectivas: la primera en la preparación de las herramientas a utilizar en la sesión de clase, éstas tienen que buscar competencias ideales en los estudiantes que fomenten la metacognición, y por otro lado permitir una reflexión constante en el educador que mejore su propia praxis. En segundo lugar está, el acercamiento continuo entre la tecnología y la educación, aspecto que constantemente sigue ganando terreno en la educación nicaragüense. 


\section{CONCLUSIONES}

La educación actual es más acertada, cuando se centra en la utilización de las nuevas tecnologías, como parte integral hacia el desarrollo de metodologías alternativas para el aprendizaje de los estudiantes.

Con la implementación de la web 2.0, el profesor ha dejado de ser el eterno conferencista y repartidor único de la información en las aulas, para convertirse en un verdadero facilitador de los aprendizajes, acorde a la globalización tecnológica de esta era.

Los estudiantes valoran de forma positiva, el uso e implementación de la web 2.0 como una herramienta pedagógica en los estudios universitarios, la innovación al usar Facebook con fines educativos, Dropbox como forma de intercambio de información académica, los tutoriales como medio didáctico en la retroalimentación de los aprendizajes y los software, como alternativa para calcular datos estadísticos que se utilizan en la disciplina investigación aplicada.

\section{REFERENCIAS}

Barajas, F. Álvarez, C. (2013). Uso de Facebook como herramienta en la enseñanza del área de naturales en el grado undécimo de educación media vocacional [Versión electrónica].Pixel-Bit. Revista de medios y educación (42), 143-156.

Cook, T. Retechardt, C. (2004). "Métodos Cuantitativos y Cualitativos en investigación Educativa”. Madrid: Morata.

Flores, J. (2014). Mateayuda: una herramienta tecnológica para reforzar aprendizajes matemáticos. [Versión electrónica]. Revista UNAN-FAREM Estelí. 3(10), 49-65.

George, D., \& Mallery, P. (2003). SPSS for Windows step by step: A simple guide and reference. 11.0 update (4th ed.). Boston: Allyn \& Bacon

Hernández, R. Fernández, C. Baptista, P. (2010). Metodología de la investigación. México: McGraw-Hill.

López, F. (2007). Metodología Participativa en la Enseñanza Universitaria. Madrid: Narcea. 1519

Zamarrazo, J. Amorós, L. (2011). Las nuevas Tecnologías de la enseñanza de las Ciencias. Sevilla: Editorial MAD. 171-172 